\title{
ARTICLES
}

\section{Macrophage Preconditioning with Synthetic Malaria Pigment Reduces Cytokine Production via Heme Iron-Dependent Oxidative Stress}

\author{
Donatella Taramelli, Stefania Recalcati, Nicoletta Basilico, Piero Olliaro, and \\ Gaetano Cairo
}

Istituto di Microbiologia (DT, NB), Università di Milano, and Istituto di Scienze Mediche (SR), IRCCS Ospedale Maggiore, Università di Milano, Milan, Italy; UNDP/WorldBank/WHO Special Programme for Research and Training in Tropical Diseases (PO), Geneva, Switzerland; and Istituto di Patologia Generale (GC), Università di Milano e Centro di Studio sulla Patologia Cellulare, Milan, Italy

SUMMARY: Hemozoin (malaria pigment), a polymer of hematin (ferri-protoporphyrin IX) derived from hemoglobin ingested by intraerythrocytic plasmodia, modulates cytokine production by phagocytes. Mouse peritoneal macrophages (PM) fed with synthetic $\beta$-hematin $(\mathrm{BH})$, structurally identical to native hemozoin, no longer produce tumor necrosis factor $\alpha$ (TNF $\alpha$ ) and nitric oxide (NO) in response to lipopolysaccharide (LPS). Impairment of NO synthesis is due to inhibition of inducible nitric oxide synthase (iNOS) production. BH-mediated inhibition of PM functions cannot be ascribed to iron release from $\mathrm{BH}$ because neither prevention by iron chelators nor down-regulation of iron-regulatory protein activity was detected. Inhibition appears to be related to pigment-induced oxidative stress because (a) thiol compounds partially restored PM functions, (b) heme oxygenase (HO-1) and catalase mRNA levels were up-regulated, and (c) free radicals production increased in $\mathrm{BH}$-treated cells. The antioxidant defenses of the cells determine the response to $\mathrm{BH}$ : microglia cells, which show a lower extent of induction of $\mathrm{HO}-1$ and catalase mRNAs and lower accumulation of oxygen radicals, are less sensitive to the inhibitory effect of $\mathrm{BH}$ on cytokine production. Results indicate that $\mathrm{BH}$ is resistant to degradation by $\mathrm{HO}-1$ and that heme-iron mediated oxidative stress may contribute to malaria-induced immunosuppression. This study may help correlate the different clinical manifestations of malaria, ranging from uncomplicated to severe disease, with dysregulation of phagocyte functions and promote better therapeutic strategies to counteract the effects of hemozoin accumulation. (Lab Invest 2000, 80:1781-1788).

\begin{abstract}
I ntraerythrocytic stage plasmodia digest hemoglobin in the food vacuole, where the globin is degraded and heme is detoxified, principally by polymerization/crystallization into hemozoin, an insoluble, microcrystalline derivative of ferri-protoporphyrin IX $(\alpha$ hematin, AH) (Bohle et al, 1997; Francis et al, 1997; Pagola et al, 2000; Slater et al, 1991). As parasites mature, hemozoin (malaria pigment) accumulates in the food vacuole and remains in the "residual body"
\end{abstract}

Received April 17, 2000

This work was supported in part by UNDP/World Bank/WHO Special Programme for Research and Training on Tropical Diseases, Geneva, $(\mathrm{CH})$ N. 97-0115/98-0363, by the Consiglio Nazionale delle Ricerche, and by the Ministero Italiano dell'Università e della Ricerca Scientifica e Tecnologica, Co-finanziamento 1999, Projects: "Molecular, immunological, and pharmacological analysis of the interactions between parasite, hosts, and vectors" to DT and "Role of iron in oxidative damage: From basic mechanisms to disease and protective strategies" to GC.

Address reprint requests to: Prof. Donatella Taramelli, Istituto di Microbiologia-Universita' di Milano, Via Pascal 36, I-20133 Milan, Italy. E-mail:donatella.taramelli@unimi.it after schizont rupture. Pigment is found inside host's phagocytes following ingestion of whole parasites or "residual bodies." Although a clinical correlation between the presence of pigment in circulating neutrophils and monocytes and disease severity is established (Nguyen et al, 1995), the nature of the interaction between pigment and phagocytes, as well as its contribution to the pathogenesis of malaria, and particularly its severe complications, are still controversial. Indeed, both pro-inflammatory (induction of tumor necrosis factor $\alpha$ [TNF $\alpha$ ], interleukin-6 [IL-6], and chemotactic cytokines) (Pichyangkul et al, 1994; Prada et al, 1995; Sherry et al, 1995) and antiinflammatory activities (inhibition of respiratory burst and adhesion molecule expression and of TNF $\alpha$ and nitric oxide [NO] production) (Prada et al, 1995; Schwarzer et al, 1992; Taramelli et al, 1995, 1998) have been described in pigment-fed phagocytes. The study of such interactions is simplified now by the availability of $\beta$-hematin $(\mathrm{BH})$, a synthetic polymer obtained from hematin in acidic conditions. Synthetic pigment is identical to the heme portion of native 
hemozoin, as conclusively demonstrated by experiments using X-ray diffraction and Fourier transforminfrared (FT-IR) spectroscopy (Bohle et al, 1997; Pagola et al, 2000; Slater et al, 1991).

Iron metabolism and macrophage functions are closely intertwined. A major role in this regulatory connection is played by the interaction of nitric oxide (NO) and iron regulatory proteins (IRP), the cytosolic proteins that control intracellular iron homeostasis by post-transcriptional regulation of ferritin and transferrin receptor expression (Hentze and Kuhn, 1996). IRP activity is mainly regulated by cellular iron levels but is also influenced by other stimuli, such as oxygen and nitrogen radicals. Indeed, we have shown that NO modulates IRP binding activity both in human (Recalcati et al, 1998a) and mouse macrophages (Recalcati et al, 1998b), thus affecting iron availability. In turn, iron levels modulate cytokine-induced transcription of inducible nitric oxide synthase (iNOS), the inducible enzyme that catalyzes NO formation (Weiss et al, 1995), thus establishing an autoregulatory loop between iron metabolism and the NO pathway in macrophages. Given this interplay between iron homeostasis and the immune function of phagocytes, we set out to assess the role of pigment heme iron in the unresponsiveness of phagocytic cells.

\section{Results}

\section{Role of Heme Iron on BH-Mediated Inhibition of Macrophage Functions}

Sonicated $\mathrm{BH}$ particles were readily phagocytized by mouse peritoneal macrophages (PM) and BV2 microglia cells. As previously reported (Taramelli et al, 1995), more than $80 \%$ PM or $85 \%$ BV2 cells were engulfed with black crystals, which by electron microscopy appeared either scattered throughout the cytoplasm or contained in phagosomes (Olliaro et al, 2000). No changes in viability, protein content, or respiratory capacity were detected after 24 hours treatment with $\mathrm{BH}$ or unpolymerized (monomeric) hematin $(\mathrm{AH})$ or protoporphyrin IX (PPIX, the same porphyrin, but devoid of iron) (Table 1$)$.

The production of nitrite by lipopolysaccharide (LPS)-stimulated PM was inhibited in a dose- dependent manner by pretreatment with $\mathrm{BH}$ and $\mathrm{AH}$, but not PPIX (Fig. 1A). Such effect of BH could be due either to a NO-scavenging reaction by the heme moiety or to direct inhibition of NO synthesis. To address this question, we investigated the expression of iNOS by Western blot analysis. Pretreatment of PM with $\mathrm{BH}$ prevented the increase in iNOS content induced by LPS (Fig. 1B), thus demonstrating that the impairment of NO production (reported in Fig. 1A) was due to decreased iNOS.

Because transcription of the iNOS gene is inhibited by iron (Weiss et al, 1995), we set out to investigate whether the reduction of iNOS content was related to increased intracellular iron, possibly released from the protoporphyrins (BH or $\mathrm{AH}$ ) (Schwarzer et al, 1992). For $\mathrm{BH}$, this proved not to be the case. RNA-bandshift assay (Fig. 1C) was used to measure the activity of iron-regulatory proteins (IRP-1 and IRP-2), which faithfully reflects the status of the intracellular chelatable iron pool (Hentze and Kuhn, 1996). No significant differences in the binding activity of both IRP-1 and IRP-2 were found in $\mathrm{BH}$-treated $\mathrm{PM}$, suggesting that iron is not released from the pigment, at least during the first 24 hours after ingestion. In contrast, but in agreement with previous findings (Hentze and Kuhn, 1996; Recalcati et al, 1999), a reduction of IRP activity was detected in PM treated with unpolymerized $\mathrm{AH}$. In this case, the effect could be prevented with the iron chelator desferrioxamine (DFO), whereas the addition of DFO was uneventful with $\mathrm{BH}$. Further evidence that impaired NO production was not caused by iron release was provided by the fact that DFO did not overcome the dose-dependent inhibition of NO production in BH-fed, LPS-stimulated PM (Fig. 1D). In the same experimental conditions, $\mathrm{TNF} \alpha$ production too was inhibited by $\mathrm{BH}$ (data not shown).

\section{BH-Induced Oxidative Stress Modulates NO Production in Mouse Macrophages and Microglia Cells}

Inhibition of NO and TNF $\alpha$ production is counterbalanced by sulfydryl group donors (Fig. 1D) (see also Taramelli et al, 1995). Therefore, we investigated whether the accumulation of undigested $\mathrm{BH}$ could trigger oxidative stress by measuring intracellular ox-

Table 1. Respiratory Activity and Protein Content of Peritoneal Macrophages from CD1 Mice and BV2 Microglia Cells Exposed to Different Stimuli

\begin{tabular}{|c|c|c|c|c|}
\hline \multirow[b]{2}{*}{ Treatment } & \multicolumn{2}{|c|}{ Peritoneal macrophages } & \multicolumn{2}{|c|}{ BV2 microglia cells } \\
\hline & $\begin{array}{c}\mathrm{MTT}^{a}(\mathrm{OD} \\
550 / 650)\end{array}$ & $\begin{array}{l}\text { Protein }^{b} \\
\mathrm{mg} / \text { well }\end{array}$ & $\begin{array}{l}\text { MTT (OD } \\
550 / 650)\end{array}$ & $\begin{array}{l}\text { Protein } \\
\text { mg/well }\end{array}$ \\
\hline Medium $^{c}$ & $0.142 \pm 0.07$ & $404 \pm 41$ & $0.466 \pm 0.03$ & $405 \pm 33$ \\
\hline BH $100 \mu \mathrm{g} / \mathrm{ml}$ & $0.108 \pm 0.21$ & $407 \pm 38$ & $0.143 \pm 0.09$ & $421 \pm 54$ \\
\hline AH $100 \mu \mathrm{g} / \mathrm{ml}$ & $0.214 \pm 0.11$ & $380 \pm 48$ & $0.711 \pm 0.12$ & $418 \pm 45$ \\
\hline PPIX $100 \mu \mathrm{g} / \mathrm{ml}$ & $0.102 \pm 0.22$ & $396 \pm 36$ & ND & $450 \pm 37$ \\
\hline
\end{tabular}

MTT, 3-(4,5-dimethylthiazol-2-yl)-2,5 diphenil tetrazolium bromide; ND, not determined.

${ }^{a}$ Assessment of respiratory activity (reduction of MTT) in peritoneal macrophages vs BV2 microglia cells treated for 24 hours with different concentrations of $\beta$ hematin $(\mathrm{BH})$ or hematin $(\mathrm{AH})$ or protoporphyrin IX (PPIX).

${ }^{b}$ The protein content per well was determined by the method of Bradford.

${ }^{c}$ Medium control with no $\beta$ hematin or hematin. 
A)
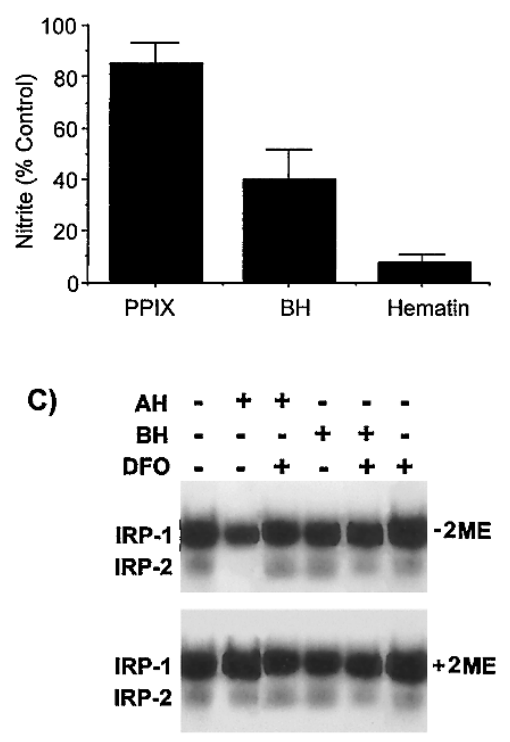

B)

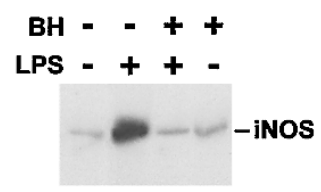

D)

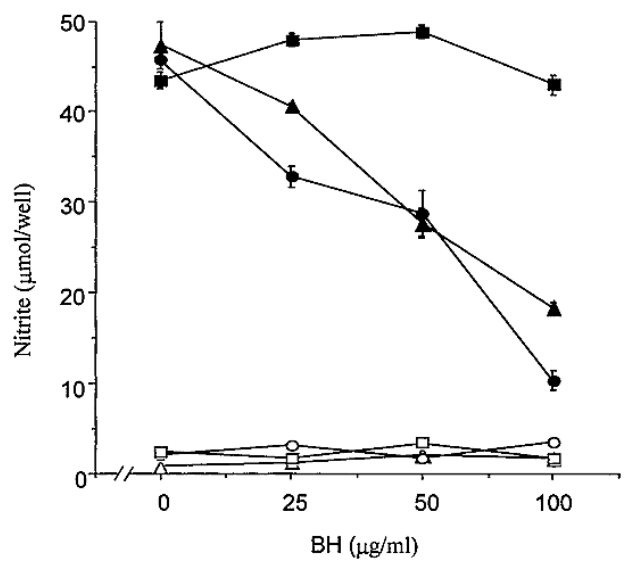

Figure 1.

Free iron does not mediate the unresponsiveness of $\beta$ hematin $(\mathrm{BH})$-treated macrophages to lipopolysaccharide (LPS). $A$, Inhibition of nitrite production in LPS-stimulated peritoneal macrophages (PM) by BH or monomeric hematin, but not non-iron protoporphyrin IX (PPIX). PM were left untreated (controls) or pretreated for 24 hours with $100 \mu \mathrm{g} / \mathrm{ml}$ of BH, $\alpha$ hematin (AH), or PPIX and then stimulated with LPS (1 $\mu \mathrm{g} / \mathrm{ml})$ for 24 hours. Data are expressed as the percentage of control \pm SD of three independent experiments, in triplicate. $B$, Western blot analysis of inducible nitric oxide synthase (iNOS) content. Cytoplasmic extracts were prepared from PM pretreated with BH and then stimulated with LPS as described above. Equal amounts of proteins were processed as described in the "Materials and Methods" section, and iNOS-corresponding bands were visualized by chemiluminescence. The results shown are representative of three independent experiments. $C$, Modulation of iron regulatory protein (IRP)-1 and IRP-2 activity in BH- or AH-treated PM, plus or minus desferrioxamine (DF0). The assay was carried out in the absence or in the presence of $2 \%$ 2-mercaptoethanol (2ME) to reveal the spontaneous and total IRP activity, respectively. The autoradiogram shown is representative of four independent experiments. $D$, Thiols, but not iron chelators, counterbalance the inhibition of nitrite production in PM. PM were pretreated for 24 hours with different doses of $\mathrm{BH}$ in the absence (black circles) or presence of $8 \mathrm{~mm}$ reduced glutathione (GSH, black squares) or $100 \mu \mathrm{m}$ DFO (black triangles) and then stimulated with LPS $(1 \mu \mathrm{g} / \mathrm{ml})$ for 24 hours. White symbols represent control supernatants of PM not stimulated with LPS. Data represent mean \pm sD from triplicate samples $(n=4)$.

ygen radicals based on quantification of the oxidationactivated fluorescent dye, 2', 7'-dichlorofluorescein diacetate (DCF-DA) (Poss and Tonegawa, 1998). A dose-dependent increase in relative fluorescence in the supernatants of $\mathrm{BH}$-treated $\mathrm{PM}$ was seen after both 4 hours (data not shown) and 24 hours (Fig. 2A). The response to unpolymerized $\mathrm{AH}$ was twice as high as with $\mathrm{BH}$, whereas no activation occurred with the non-iron-containing PPIX. In addition, we measured by Northern blot analysis the amount of two transcripts involved in the oxidative stress response: heme-oxygenase $1(\mathrm{HO}-1)$, a sensitive marker of oxidative stress (Poss and Tonegawa, 1997), and catalase, an enzyme involved in hydrogen peroxide catabolism. HO-1 mRNA levels increased 4 hours after $\mathrm{BH}$ treatment (Fig. 2B), albeit comparatively less than after treatment with unpolymerized $\mathrm{AH}$, the physiologic inducer of HO-1 (4.8- and 8.3-fold over control levels, respectively). Induction of catalase gene expression was lower (1.8- and 2.6-fold by $\mathrm{BH}$ and $\mathrm{AH}$, respectively). In this case too, PPIX did not show appreciable effects on the level of either transcript, thus confirming the need of iron for activation.

In contrast, in BV2 microglia cells, HO-1 mRNA accumulation increased 3.2-fold with hematin and 1.9-fold with $\mathrm{BH}$, whereas catalase gene expression was slightly enhanced (1.7-fold) only by $\mathrm{AH}$ (Fig. 3A). Similarly, both $\mathrm{BH}$ and $\mathrm{AH}$ induced the release of oxygen radicals (Fig. 3B), but the fluorescence intensity was considerably lower than in PM (compare Figs. $2 \mathrm{~A}$ and $3 \mathrm{~B}$ ). Because the two cell types ingested $\mathrm{BH}$ to a similar extent (Taramelli et al, 1995), the lower response to oxidant stress cannot be attributed to a different accumulation of pigment and is likely to depend on the cell's antioxidant status. In fact, BV2 cells have a lower content of unsaturated membrane fatty acids and higher levels of reduced glutathione (GSH) levels, both indicative of reduced sensitivity to oxidant stress (Omodeo-Salè et al, 1998).

\section{Discussion}

The results of this study demonstrate that the ingestion of synthetic malaria pigment, $\mathrm{BH}$, triggers oxidative stress, which in turn induces depression of phagocyte functions. Moreover, they provide evidence of the relationship between iron, oxidative stress, and immune response in malaria. These results are consistent with the iron-mediated inhibition of iNOS transcription (Weiss et al, 1995) and the NO-dependent, IRP-mediated modulation of the expression of iron genes (Recalcati et al, 1998a, 1998b). In the case of 
A)

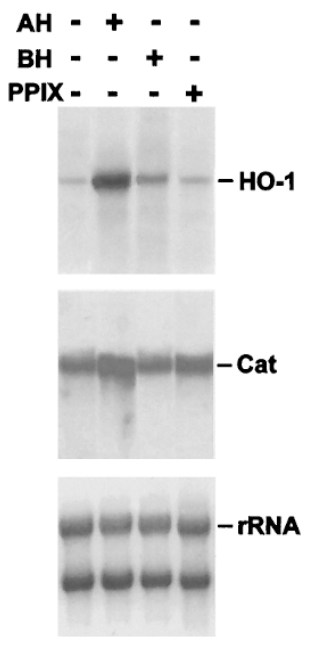

B)

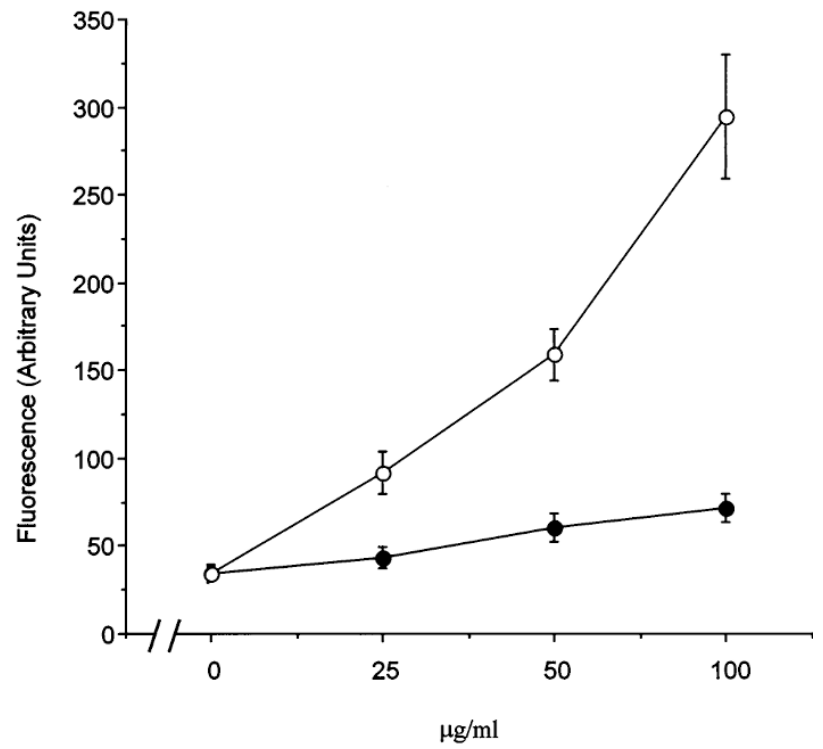

Figure 3.

Free radicals production and induction of stress protein mRNAs in BV2 microglia cells treated with $\mathrm{BH}$. A, Northern blot analysis of $\mathrm{HO}-1$ and Cat mRNA of BV2 microglia cells treated for 4 hours with $\mathrm{BH}, \mathrm{AH}$, or PPIX. Total RNA was extracted, Northern-blotted, and hybridized as described in the legend to Figure 2. The autoradiograms shown are representative of three independent experiments. $B$, Extracellular DCF-DA fluorescence was recorded after 24 hours incubation of BV2 cells with different doses of $\mathrm{BH}$ (black circles) or $\mathrm{AH}$ (white circles). Cell loading with $20 \mu \mathrm{m}$ DCF-DA was performed for 15 minutes before the addition of the stimuli. Data show mean \pm SEM.

the unresponsiveness of malaria pigment-fed macrophages, we demonstrate that the metal does not act directly, but rather as a catalyst of oxidative damage. This conclusion is based on several observations: (a) the ingestion of $\mathrm{BH}$ triggers the production of reactive oxygen radicals and the up-regulation of stress proteins such as $\mathrm{HO}-1$ and catalase; (b) $\mathrm{BH}$-fed phagocytes recover the ability to produce cytokines upon addition of sulfydryl-group donors; (c) heme-bound, but not free, iron is required; and (d) pigment effects depend on a cell's susceptibility to oxidative stress.

Because reduced glutathione is critical to iNOS mRNA induction (Duval et al, 1995), the oxidantdependent depletion of intracellular thiol levels could represent the molecular connection between oxidative stress and the inhibition of iNOS synthesis. A direct inhibition of iNOS by hydrogen peroxide has also been reported in mesangial cells (Jaimes et al, 1997). Furthermore, prior induction of a stress response has been shown to inhibit nuclear transcription factor $\kappa \mathrm{B}$ $(\mathrm{NF}-\kappa \mathrm{B})-$ mediated gene regulation (Wong et al, 1997) and cytokine release (Zahler et al, 2000). Thus, BHinduced production of oxygen free radicals may prevent iNOS gene transcription, possibly through inhibition of protein kinase $\mathrm{C}$ membrane translocation (Arese and Schwarzer, 1997), by blocking the interaction of NF- $\kappa \mathrm{B}$ with its binding site in the $5^{\prime}$-flanking region of the iNOS gene.

Alternatively, the effect of oxidative stress on the impairment of PM functions could be mediated not by oxygen radicals per se, but through $\mathrm{HO}-1$ induction. Both a pro- and anti-inflammatory role have been ascribed to HO-1. In particular, our results point to the existence of an immunoregulatory loop involving undegraded heme/malaria pigment, $\mathrm{HO}-1$ up-regulation, and phagocytes functions. This immunoregulatory loop may be relevant in the pathogenesis of malaria infections. HO-1 is induced by a variety of agents causing oxidative stress (Poss and Tonegawa, 1998), but also undergoes adaptive regulation in response to its physiological substrate, heme. Although we cannot rule out the possibility that the increased $\mathrm{HO}-1$ expression may be a direct effect of the heme moiety of $\mathrm{BH}$, the results presented in Figure 2, along with the finding that the effect of $\mathrm{BH}$ on nitrite production was prevented by reducing agents, suggest that $\mathrm{HO}-1$ up-regulation is caused by $\mathrm{BH}$-induced oxidative injury. This conclusion is in agreement with the increased levels of lipid peroxidation induced by malaria pigment in human monocytes (Arese and Schwarzer, 1997) and mouse macrophages (Omodeo-Salè et al, 1998). In view of these considerations, it is difficult to reconcile our results with recent reports of the lack of induction of HO-1 in hemozoin-fed human monocytes (Schwarzer et al, 1999) that had been shown to experience oxidative stress upon ingestion of hemozoin (Schwarzer et al, 1992). Whether $\mathrm{BH}$ and native hemozoin indeed differ in their ability to induce $\mathrm{HO}-1$ expression in different cells is presently under investigation. Undeniably, differences, if they exist, may not be ascribed to the hematin polymer per se (that is identical in the native and natural compound), but rather to the lipid and/or protein components of the pigment. Results with the latter compound are, however, largely determined by the method of preparation (Pichyangkul et al, 1994). 
A)

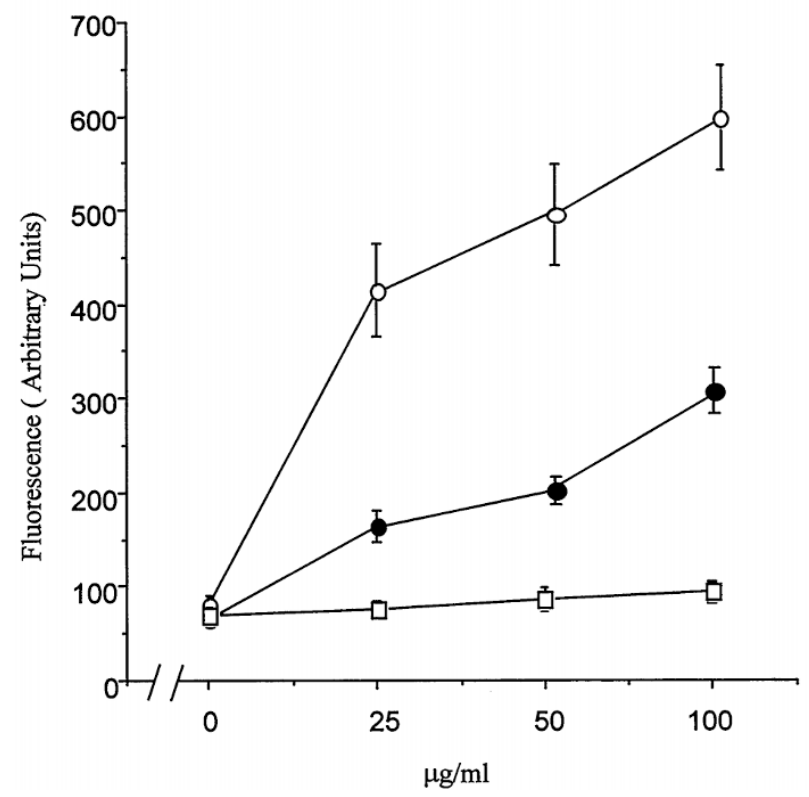

B)

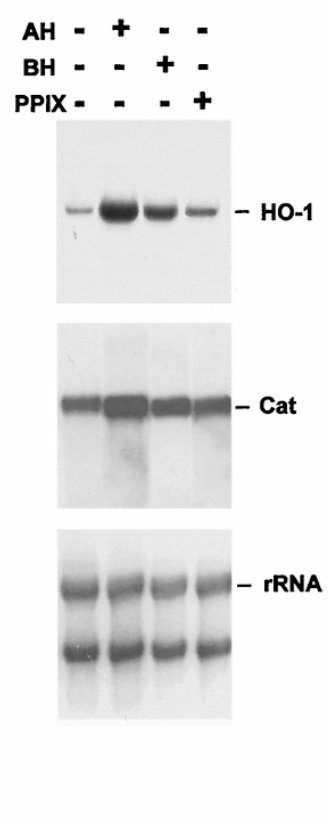

Figure 2.

Free radicals production and induction of stress protein mRNAs in BH-fed PM. A, Extracellular 2', 7'-dichlorofluorescein diacetate (DCF-DA) fluorescence was recorded after 24 hours incubation of PM with different doses of $\mathrm{BH}$ (black circles), monomeric $\mathrm{AH}$ (white circles), or PPIX (white squares). Cell loading with $20 \mu \mathrm{M}$ DCF-DA was performed for 15 minutes before the addition of the stimuli. Data show mean $\pm \mathrm{SEM}$. $B$, Northern blot analysis of heme oxygenase (HO-1) and catalase (Cat) mRNA levels of PM treated for 4 hours with BH, AH, or PPIX. Total RNA was extracted, Northern-blotted, and hybridized with ${ }^{32} \mathrm{P}$-labeled rat H0-1 and rat catalase probes. The autoradiograms shown are representative of three independent experiments.

Indeed, the anti-inflammatory role of $\mathrm{HO}-1$ has been recently largely documented: (a) $\mathrm{HO}-1$ up-regulation and inflammation are inversely related (Foresti and Motterlini, 1999; Willis et al, 1996); (b) HO-1-deficient mice present symptoms similar to those of patients with chronic inflammation (Poss and Tonegawa, 1997); (c) HO-1 may have a protective effect against arteriosclerosis associated with chronic rejection of transplants (Hancock et al, 1998) and against ischemia/reperfusion injury in a rat model (Amersi et al, 1999). Moreover, the end products of $\mathrm{HO}-1$ activation, including biliverdin, bilirubin, and $\mathrm{CO}$, are potent antioxidants and are known to modulate immune effector functions. In particular, CO directly inhibits iNOS activity by binding to the heme moiety of iNOS and thus down-regulating NO production (Ryter and Tyrrel, 2000).

The finding that oxidative stress-mediated activation of $\mathrm{HO}-1$ in pigment-fed macrophages prevents the subsequent inflammatory response is in line with the above observations. On the other hand, lack of down-regulation of $\mathrm{NO}$ and $\operatorname{TNF} \alpha$ production by stress-resistant microglia cells may contribute to the deleterious and abnormal cytokine levels associated with cerebral malaria (Grau et al, 1987; Kremsner et al, 1996; Kwiatkowski et al, 1990; Newton at al, 1998), leading to the apparently paradoxical and unexpected conclusion that oxidative stress may be beneficial in some anatomical districts. This iterates the views cautioning against labeling biological processes as exclusively favorable or exclusively noxious (Platt and Nath, 1998).

The induction of $\mathrm{HO}-1$ by $\mathrm{BH}$ does not lead to an increase of intracellular iron levels, suggesting that, differently from monomeric hematin, $\mathrm{BH}$ is not readily susceptible to enzymatic cleavage by $\mathrm{HO}-1$ and remains apparently undegraded inside the phagocytes. This is in agreement with similar findings with native hemozoin in monocytes (Schwarzer et al, 1999).

The lack of IRP down-regulation, as well as the observation that the treatment of $\mathrm{BH}$-fed macrophages with DFO does not lead to functional recovery, allows hypothesizing that, albeit inhibition of $\mathrm{NO}$ and TNF $\alpha$ production requires iron, the effect occurs while the metal is porphyrin-bound. This contrasts with previous work (Schwarzer et al, 1992) ascribing intoxication of human monocytes to iron released from ingested hemozoin (resulting in approximately $120 \mu \mathrm{m}$ of free intracellular iron). This could not be the case because the release of such an amount of iron would be easily detected by the IRP bandshift assay which can measure variations of the free iron pool in the nanomolar range (Pourzand et al, 1999). In principle, we cannot exclude the possibility that the unchanged IRP activity in $\mathrm{BH}$-treated cells may be the net result of the opposed effects of iron-mediated down-regulation and $\mathrm{H}_{2} \mathrm{O}_{2}$-mediated up-regulation. However, we believe there is sufficient convincing evidence that this is not the case: (a) IRP-2 activity, which is sensitive to iron (Hentze and Kuhn, 1996) but not to $\mathrm{H}_{2} \mathrm{O}_{2}$ (Menotti 
et al, 1998, and references therein), is also unchanged in $\mathrm{BH}$-treated cells, and (b) IRP activity is clearly down-regulated in macrophages treated with unpolymerized $\mathrm{AH}$ in which production of oxidant species detected by DCF-DA is even greater than in $\mathrm{BH}$ treated cells (Fig. 2A).

The occurrence of oxidative stress in the absence of detectable iron mobilization may involve the formation of higher oxidation states of iron, which remain coordinated within the porphyrin molecule and cannot be intercepted by chelators. This bears similarities with the oxoferryl moiety of activated peroxidases (Kim and Sevanian, 1991). Whether the polymerization of hematin into hemozoin or $\mathrm{BH}$ enhances the formation and half-life of peroxidase-like species remains to be established. The mechanism proposed above would account for several aspects of the oxidative potential of malaria pigment, including the lack of protection by DFO as opposed to protection by electron donors like thiols or Trolox. From a therapeutic viewpoint, the results obtained with $\mathrm{BH}$-fed $\mathrm{PM}$ would provide alternatives to the minor improvements obtained with iron chelators in cerebral malaria (Weiss et al, 1998) or the recrudescence of parasitemia after treatment (Gordeux et al, 1992). They may also help provide a rationale for protective interventions with anti-oxidants.

\section{Conclusions}

These findings potentially have important clinical and pathophysiological correlates and may help reconcile reports of both pro-inflammatory (Pichyangkul et al, 1994; Prada et al, 1995; Sherry at al, 1995) and suppressive (Arese and Schwarzer, 1997; Schwarzer et al, 1992; Taramelli et al, 1995, 1998) activity of ingested hemozoin in either human or mouse phagocytes. The risk for malaria-associated morbidity and mortality is highest in nonimmune subjects, particularly young children living in endemic areas. In these subjects, severity of disease correlates, amongst other things, with the levels of TNF $\alpha$ (Grau et al, 1987; Kwiatkowski et al, 1990; Newton et al, 1998) and pigment load in circulating monocytes and neutrophils (Nguyen et al, 1995). As patient conditions deteriorate and cerebral malaria occurs, brain capillaries are filled with infected erythrocytes and pigment-laden monocytes (Newton et al, 1998). It is conceivable that a vicious circle occurs whereby increasing oxidative damage is induced by pigment that continues to accumulate in increasing numbers of sequestered phagocytes. All cells are not equally susceptible to oxidative damage, thus it should be possible to predict the resulting effect of pigment in different tissues, ie, accumulation in stress-resistant tissues would prolong and exacerbate the inflammatory response. With age, immunity builds up in areas of intense transmission where repeat exposure to malaria infection consorts with accumulating pigment, thus unwavering induction of $\mathrm{HO}-1$, which may play an antiinflammatory role. Cellular and humoral immunity, as well as down-regulation of excessive release of medi- ators, may contribute to the milder manifestations of malaria in adults from endemic areas.

\section{Methods}

\section{Reagents}

Unless otherwise indicated, all reagents were obtained from Sigma Italia (Milan, Italy).

\section{Synthesis of $\beta$-Hematin}

$\mathrm{BH}$ was synthesized according to Slater et al (1991) with slight modifications. In short, $60 \mu$ moles of hematin (ferri-protoporphyrin IX hydroxide) were dissolved in $8 \mathrm{ml}$ of $0.1 \mathrm{M} \mathrm{NaOH}$, under $\mathrm{N}_{2}$, then precipitated by the addition of $49 \mathrm{mmol}$ of acetic acid. The suspension was heated overnight at $70^{\circ} \mathrm{C}$, and the precipitate was then centrifuged and washed four times with distilled water. Unreacted hematin was removed by extracting the precipitate twice for 3 hours in $0.1 \mathrm{M}$ sodium bicarbonate buffer at $\mathrm{pH} 9.1$. The remaining insoluble material was recovered by centrifugation, washed four times in distilled water, and lyophilized. The characteristics of the final product were routinely controlled by infrared spectroscopy as reported elsewhere (Slater et al, 1991). Batches of $\mathrm{BH}$ that did not meet standard criteria for purity were discarded. To facilitate the treatment of cell monolayers with insoluble $\mathrm{BH}$, the compound was resuspended in the culture medium, mechanically microdispersed, and then sonicated (Taramelli et al, 1995).

\section{Cell Isolation and Treatment}

Proteose-peptone-elicited peritoneal macrophages (PM) were recovered from 6- to 8-week-old, pathogen-free female CD1 mice (Charles River Italia, Calco, Italy) that were housed, fed, and handled in compliance with the prescriptions for the care and use of laboratory animals. PM were purified by adherence to plastic in tissue culture clusters (Corning-Costar Italia, Milan, Italy) for 2 hours at $37^{\circ} \mathrm{C}$ in $5 \% \mathrm{CO}_{2}$. The retrovirus immortalized, BV2 microglia cell line of Balb/c origin (Blasi et al, 1990), was kindly provided by Prof. Elisabetta Blasi, University of Modena, Italy. Cells were grown in RPMI 1640 (HyClone Laboratories, Logan, Utah) supplemented with $100 \mathrm{IU} / \mathrm{ml}$ of penicillin, $2 \mathrm{~mm}$ glutamine, $100 \mu \mathrm{g} / \mathrm{ml}$ streptomycin, 20 mM Hepes buffer, and 5\% FCS (HyClone). PM and BV2 cells were seeded in quadruplicate and treated with $\mathrm{BH}$ or freshly prepared hematin $(\mathrm{AH})$ or protoporphyrin IX for different times. Cell viability and metabolic activity were tested by trypan blue exclusion and reduction of 3-(4, 5-dimethylthiazol-2-yl)-2,5 diphenil tetrazolium bromide (MTT) to formazan crystals, as described (Taramelli et al, 1995). The plates were then read on a microplate reader (Molecular Devices Company, Menlo Park, California) using a test wavelength of $550 \mathrm{~nm}$ and a reference wavelength of $680 \mathrm{~nm}$. Where indicated, reduced glutathione (GSH), desferrioxamine (DFO), or Trolox C were used together with $\mathrm{BH}$ to treat $\mathrm{PM}$ or $\mathrm{BV} 2$ cells. For the detection of 
oxidative stress, DCF-DA (Sigma Italia) was freshly diluted in culture medium from stock solutions in methanol and added to PM or BV2 cultures at $50 \mu \mathrm{M}$ for 15 minutes. Cells were then washed and incubated overnight with the appropriate stimulus at $37^{\circ} \mathrm{C}$. The fluorescence of the supernatants was determined using a Perkin Elmer LS50 luminescence spectrometer (Perkin Elmer, Foster City, California) with an excitation wavelength of $492 \mathrm{~nm}$ and emission at $522 \mathrm{~nm}$ (Poss and Tonegawa, 1998). Data were processed using the Perkin Elmer data manager. The Griess reagent was used to measure nitrites in the supernatants of macrophages stimulated with LPS (1 $\mu \mathrm{g} / \mathrm{ml})$ for 24 hours (Taramelli et al, 1995).

\section{RNA-Protein Gel Retardation Assay}

Cell lysates were prepared from PM and incubated in the absence or presence of $2 \% 2$-mercaptoethanol, with a molar excess of a probe transcribed from linearized pSPT-fer, which contains the iron responsive elements (IRE) of human ferritin $\mathrm{H}$ chain, as described previously (Cairo et al, 1997). After separation on $6 \%$ nondenaturing polyacrylamide gels, RNAprotein complexes were visualized by autoradiography and quantified by direct nuclear counting using an Instant Imager (Packard Instrument Company, Milano, Italy).

\section{Western Blot Analysis}

Equal amounts of proteins from PM cytosolic extracts were electrophoresed in 10\% acrylamide-SDS gels, electroblotted to Hybond PVDF membranes (Amersham Pharmacia Biotech, Milan, Italy), and incubated with a 1:1.000 dilution of antiserum to mouse iNOS (Alexis Corporation, Läufelfingen, Switzerland). iNOS was detected by chemiluminescence using an immunodetection kit (ECL Plus, Amersham Pharmacia Biotech) according to the manufacturer's instructions (Recalcati et al, 1998a).

\section{Northern Blot Analysis}

Total cellular RNA was isolated as described (Cairo et al, 1995) and equal amounts of RNA were electrophoresed under denaturing conditions. To confirm that each lane contained equal amounts of total RNA, the ribosomal RNA content in each lane was estimated in the ethidium bromide-stained gels by laser densitometry. RNA was transferred to Hybond-N filters (Amersham Corporation) that were hybridized with the ${ }^{32} \mathrm{P}$ labeled rat pRHO1 heme oxygenase cDNA (Cario et al, 1995) and rat pMJ512 catalase cDNA (Furuta et al, 1986). Quantitative determination was achieved by direct nuclear counting using an Instant Imager (Packard Instrument Company) and the values were normalized to the amount of ribosomal RNA.

\section{Acknowledgements}

The authors thank Prof. G. Minotti, Università di Chieti, Prof. F. Omodeo-Salè, Universita' di Milano, and Dr. D. Monti, CSSON-CNR, Milan, Italy, for helpful discussions and critical reading of the manuscript.

\section{References}

Amersi F, Buelow R, and Kato $H$ (1999). Upregulation of heme oxygenase-1 protects genetically fat Zucker rat livers from ischemia/reperfusion injury. J Clin Invest 104:16311639.

Arese P and Schwarzer E (1997). Malarial pigment (haemozoin): A very active "inert" substance. Ann Trop Med Parasitol 91:501-516.

Blasi E, Barluzzi R, Bocchini V, Mazzolla R, and Bistoni F (1990). Immortalization of murine microglial cells by a v-raf/ v-myc carrying retrovirus. J Neuroimmunol 27:229-237.

Bohle SD, Dinnebier RE, Madsen SK, and Stephens PW (1997). Characterization of the products of the heme detoxification pathway in malarial late trophozoites by X-ray diffraction J Biol Chem 272:713-716.

Cairo G, Recalcati S, Montosi G, Castrusini E, Conte D, and Pietrangelo A (1997). Inappropriately high iron regulatory protein reactivity in monocytes of patients with genetic homochromatosis. Blood 89:2546-2549.

Cairo G, Tacchini L, Pogliaghi G, Anzon E, Tomasi A, and Bernelli-Zazzera A (1995). Induction of ferritin synthesis by oxidative stress: Transcriptional and posttranscriptional regulation by expansion of the "free" iron pool. J Biol Chem 270:700-703.

Duval DL, Sieg DJ, and Billings RE (1995). Regulation of hepatic nitric oxide synthase by reactive oxygen intermediates and glutathione. Arch Biochem Biophys 316:699-706.

Foresti R and Motterlini R (1999). The heme oxygenase pathway and its interaction with nitric oxide in the control of cellular homeostasis. Free Radic Res 31:459-475.

Francis SE, Sullivan DJ, and Goldberg DE (1997). Hemoglobin metabolism in the malaria parasite Plasmodium falciparum. Annu Rev Microbiol 51:97-123.

Furuta S., Hayashi H, Miyazawa S, Osumi T, and Hashimoto T (1986). Complete nucleotide sequence of cDNA and deduced amino acid sequence of rat liver catalase. Proc Natl Acad Sci USA 83:313-317.

Gordeux VR, Thuma PE, Brittenham GM, Zulu S, Simwanza G, Mhangu A, Flesch G, and Parry D (1992). Iron chelation with desferrioxamine $\mathrm{B}$ in adults with asymptomatic Plasmodium falciparum parasitemia. Blood 79:308-312.

Grau GE, Fajardo LF, Piguet P, Allet B, Lambert P, and Vassalli $P$ (1987). Tumor necrosis factor (cachectin) as an essential mediator in murine cerebral malaria. Science 237 : 1210-1212.

Hancock WW, Buelow R, Sayegh MH, and Turka LA (1998). Antibody-induced transplant arteriosclerosis is prevented by graft expression of anti-oxidant and anti-apoptotic genes. Nat Med 12:1392-1396.

Hentze MW and Kuhn LC (1996). Molecular control of vertebrate iron metabolism: mRNA-based regulatory circuits op- 
erated by iron, nitric oxide, and oxidative stress. Proc Natl Acad Sci USA 93:8175-8182.

Jaimes EA, Nath KA, and Raij L (1997). Hydrogen peroxide downregulates IL-1-driven mesangial iNOS activity: Implications for glomerulonephritis. Am J Physiol 272:F721-F728.

Kim EH and Sevanian A (1991). Hematin- and peroxidecatalyzed peroxidation of phospholipid liposomes. Arch Biochem Biophys 288:324-330.

Kremsner PG, Winkler S, Wildling E, Prada J, Brienzle U, Graninger W, and Nussler AK (1996). High plasma levels of nitrogen oxides are associated with severe disease and correlate with rapid parasitological and clinical cure in Plasmodium falciparum malaria. Trans $\mathrm{R}$ Soc Trop Med Hyg 90:44-47.

Kwiatkowski D, Hill AS, Sambou I, Twumasi P, Castracane J, Manogue KR, Cerami A, Brewster DR, and Greenwood BM (1990). TNF concentration in fatal cerebral, non-fatal cerebral, and uncomplicated Plasmodium falciparum malaria. Lancet 336:1201-1204.

Menotti E, Henderson BR, and Kuhn LC (1998). Translational regulation of mRNAs with distinct IRE sequences by iron regulatory proteins 1 and 2. J Biol Chem 273:1821-1824.

Newton CR, Taylor TE, and Whitten RO (1998). Pathophysiology of fatal falciparum malaria in African children. Am J Trop Med Hyg 58:673-683.

Nguyen PH, Day N, Pram TD, Ferguson DJ, and White NJ (1995). Intraleucocytic malaria pigment and prognosis in severe malaria. Trans R Soc Trop Med Hyg 89:200-204.

Olliaro P, Lombardi L, Frigerio S, Basilico N, Taramelli D, and Monti D (2000). Phagocytosis of hemozoin (native and synthetic malaria pigment), andPlasmodium falciparum intraerythrocyte-stage parasites by human and mouse phagocytes. Ultrastruct Pathol 24:9-13.

Omodeo-Salè F, Basilico N, Folini M, Olliaro P, and Taramelli D (1998). Macrophage populations of different origins have distinct susceptibilities to lipid peroxidation induced by $\beta$-haematin (malaria pigment). FEBS Lett 433:215-218.

Pagola S, Stephens PW, Bohle DS, Kosar AD, and Madsen SK (2000). The structure of malaria pigment beta-haematin. Nature 404:307-310

Pichyangkul S, Saengkrai P, and Webster HK (1994). Plasmodium falciparum pigment induces monocytes to release high levels of tumor necrosis factor- $\alpha$ and interleukin- $1 \beta$. Am J Trop Med Hyg 51:430-435.

Platt JL and Nath KA (1998). Heme oxygenase: Protective gene or Trojan horse. Nat Med 4:1364-1365.

Poss KD and Tonegawa S (1998). Heme oxygenase 1 is required for mammalian iron re-utilization. Proc Natl Acad Sci USA 94:10919-10924.

Poss KD and Tonegawa S (1997). Reduced stress defense in heme oxygenase 1-deficient cells. Proc Natl Acad Sci USA 94:10925-10930.

Pourzand C, Watkin RD, Brown JE, and Tyrrell RM (1999). Ultraviolet $A$ radiation induces immediate release of iron in human primary skin fibroblasts: The role of ferritin. Proc Natl Acad Sci USA 96:6751-6756.

Prada J, Malinowsky J, Muller S, Bienzle U, and Kremsner PG (1995). Hemozoin differentially modulates the production of interleukin 6 and tumor necrosis factor in murine malaria. Eur Cytokine Netw 6:109-112.
Recalcati S, Conte D, and Cairo G (1999). Preferential activation of iron-regulatory protein-2 in cell lines as a result of higher sensitivity to iron. Eur J Biochem 259:304-309.

Recalcati S, Pometta R, Levi S, Conte D, and Cairo G (1998a). Response of monocyte iron regulatory protein activity to inflammation: Abnormal behavior in genetic hemochromatosis. Blood 91:2565-2569.

Recalcati S, Taramelli D, Conte D, and Cairo G (1998b). Nitric oxide-mediated induction of ferritin synthesis in $\mathrm{J} 774$ macrophages by inflammatory cytokines: Role of selective iron regulatory protein-2 downregulation. Blood 91:1059-1066.

Ryter SW and Tyrrel RM (2000). The heme synthesis and degradation pathways: Role in oxidant sensitivity. Free Radic Biol Med 28:289-309.

Schwarzer E, De Matteis F, Giribaldi G, Ulliers D, Valente E, and Arese P (1999). Hemozoin stability and dormant induction of heme oxygenase in hemozoin-fed human monocytes. Mol Biochem Parasitol 100:61-72.

Schwarzer E, Turrini F, Ulliers D, Giribaldi G, Ginsburg H, and Arese $P$ (1992). Impairment of macrophage functions after ingestion of Plasmodium falciparum-infected erytrocytes or isolated malarial pigment. J Exp Med 176:1033-1041.

Sherry B, Alava G, Tracey K, Martiney J, Cerami A, and Slater A (1995). Malaria-specific metabolite hemozoin mediates the release of several potent endogenous pyrogens (TNF, MIP1 $\alpha$, and MIP-1 $\beta$ ) in vitro and altered thermoregulation in vivo. J Inflamm 45:85-96.

Slater AFG, Swiggard WJ, Orton BR, Flitter WD, Goldberg DE, Cerami A, and Henderson GB (1991). An ironcarboxylate bond links the heme units of malaria pigment. Proc Natl Acad Sci USA 88:325-329.

Taramelli D, Basilico N, De Palma AM, Saresella M, Ferrante P, Mussoni L, and Olliaro P (1998). The effect of synthetic malaria pigment (beta-hematin) on adhesion molecule expression and interleukin- 6 production by human endothelial cells. Trans R Soc Trop Med Hyg 92:57-62.

Taramelli D, Basilico N, Pagani E, Grande R, Monti D, Ghione $M$, and Olliaro P (1995). The heme moiety of malaria pigment ( $\beta$-hematin) mediates the inhibition of nitric oxide and tumor necrosis factor- $\alpha$ production by lipopolysaccharidestimulated macrophages. Exp Parasitol 81:501-511.

Weiss G, Thuma PE, Biemba G, Mazeba G, Werner ER, and Gordeux V (1998). Cerebrospinal fluid level of biopterin, nitric oxide metabolites, and immune activation markers and the clinical course of human cerebral malaria. $\mathrm{J}$ Infect Dis 177: 1064-1068.

Weiss G, Watcher H, and Fuchs D (1995). Linkage of cellmediated immunity to iron metabolism. Immunol Today 16 : 495-500.

Willis D, Moore AR, Frederick R, and Willoughby DA (1996). Heme oxygenase: A novel target for the modulation of the inflammatory response. Nat Med 2:87-90.

Wong HR, Ryan M, and Wispe JR (1997). Stress response decreases NF-kappaB nuclear translocation and increases I-kappaBalpha expression in A549 cells. J Clin Invest 99: 2423-2428.

Zahler S, Kupatt C, and Becker BF (2000). Endothelial preconditioning by transient oxidative stress reduces inflammatory responses of cultured endothelial cells to TNF $\alpha$ FASEB J 14:555-564. 